\title{
A WAY TO PREVENT THE PANDEMIC OUTBREAK OF nCOVID-19 IN INDIA
}

\author{
NIKITA PAL ${ }^{1 *}$, SHAMBADITYA GOSWAMI², RAVINDRA PAL SINGH ${ }^{3}$, PRASHANT SINGH ${ }^{3}$
}

${ }^{1}$ Department of Pharmacy Practice, NIMS Institute of Pharmacy, NIMS University, Jaipur, Rajasthan, India. ${ }^{2}$ Department of Pharmaceutical Chemistry, NIMS Institute of Pharmacy, NIMS University, Jaipur, Rajasthan, India. ${ }^{3}$ Department of Pharmaceutics, NIMS Institute of Pharmacy, NIMS University, Jaipur, Rajasthan. Email: nikitapal192@gmail.com

\author{
Received: 06 April 2020, Revised and Accepted: 29 May 2020
}

(C) 2020 The Authors. Published by Innovare Academic Sciences Pvt Ltd. This is an open access article under the CC BY license (http://creativecommons. org/licenses/by/4. 0/) DOI: http://dx.doi.org/10.22159/ajpcr.2020.v13i7.37789

\section{Dear Editor}

The recent global burden is no doubt the pandemic attack of novel coronavirus disease-19 (nCOVID-19) in the world. In India, the latest confirmed cases are 4760, recovered 349, and deceased 135 (last updated 06/04/2020; 10:16 pm) [1]. Although the death rate of nCOVID-19 is approx. 2-4\%, it is highly contagious [2]. As per the WHO, based on 44,000 patients with this disease, $81 \%$ develop mild symptoms; $14 \%$ develop severe symptoms; and 5\% undergo in critically ill condition. Coronaviruses are zoonotic, i.e., they are transmitted by animals and people. The new strain of coronaviruses is 2019-nCoV or severe acute respiratory syndrome (SARS) CoV 2, which is betacoronavirus, causes respiratory tract infection such as Middle East respiratory tract syndrome (MERS) and SARS [3]. However, $\geq 60$ years or above aged people are at high risk ( $3 \%$ ) which has a weak immune system with co-morbid conditions such as diabetes, cancer, and cardiovascular disease, but the prevalence of nCOVID-19 in young adults and adults (20-59 years) is more ( $~ 87 \%)[4]$.

The onset of disease may cause severe pneumonia and acute respiratory failure due to alveolar wall damage partially covered by epithelial cells and hyaline membranes $[5,6]$. The most common symptoms include mild fever, dry mouth, fatigue, and sore throat. In severe conditions, pleuritic chest pain has been reported. Sometimes diarrhea, nausea, vomiting may happen. Patients with older age and immunodeficiency may or may not develop fever [7].

Novel coronavirus disease is a highly transmittable droplet infection. The transmission can cause by close contact of person-to-person. Males are highly susceptible as compare to females.

Identification of SARS $\mathrm{CoV} 2$ can be done by real-time reverse transcriptase-polymerase chain reaction to take upper and lower respiratory specimen through nasopharyngeal and oropharyngeal swab as well as specimens from blood serum.

There is no specific treatment and vaccine for novel coronavirus disease till now. Only symptomatic and prophylactic therapies are given to the patient. The combination of Lopinavir-Ritonavir (double dose than HIV infection) has been approved and evidence of empirically used to treat SARS and MERS. The derivative of chloroquine and hydroxychloroquine is given by combination with azithromycin in some protocols because there is a risk of cardiac arrhythmia and in India, one death was occurred due to these drugs. As this disease attacks those people whose immune system comparatively weak, so immunomodulators such as tocilizumab (monoclonal antibody) are under investigation. Although corticosteroids are not prescribed in viral pneumonia but in some protocols, COVID-19 patients with refractory shock or acute respiratory distress, these drugs are recommended [8].

As there is no proper treatment for novel coronavirus disease till the date, social distancing and proper precautions are the only way out to control this worldwide pandemic situation. Developing countries like India, where population density is larger than life-saving equipments, early lockdown is the only sensible decision taken by the Indian Govt. Other preventive measures are:

- Frequently wash hands with soap and water in the interval of $1 / 2-1 \mathrm{~h}$. Use highly alcohol-based sanitizer until next possible hand wash

- Avoid touching nose, eyes, and face

- Stay home as much as possible. If coming from outside, put off clothes and wash face and hands thoroughly

- Get off from gathering, avoid shaking hands, and maintain a safe distance (minimum $1 \mathrm{~m}$ or 3 feet) from people

- At the time of sneezing, coughing; flexed elbow or use disposable tissues, handkerchiefs

- Use mask, gloves, and cover head

- Methylxanthines (comprise caffeine, present in tea) are nitrogencontaining compounds that act as bronchodilators mainly used to treat respiratory diseases. Hence, herbal tea with ginger and basil may be an alternative approach in this case

- Intake Vitamin C tablet with or without food and Vitamin C containing fruits and vegetables such as lemon, orange, kiwi, berries, tomatoes, sprouts, and broccoli to prevent cytokine-induced lung damage

- In case of the normal flu-like condition, take steam vapors, make gargles with antiseptics such as betadine or clove, ginger, and basil which relieve the patient by clearing throat

- To avoid dry mouth and relieve from the sore throat, frequently take lukewarm water

- As a self-checking measure, one can do Kumbhaka (retention of breath) at least for the $10 \mathrm{~s}$; during this period, if there is a pain in the chest, visit doctor immediately. There may be a chance of fibrosis in lungs

- Freehand exercises along with Ujjayi Pranayama, Simhasana, Sarvangasana to keep the body fit and enhance the immune system

- Last but not the least, minimum 10 min meditation keeps the mindbody fresh and healed.

The name CORONA itself tells to take precautions: C - Clean hands, 0 - Off from gathering, R - Raise immunity, 0 - Offer hand wash to poor people, $\mathrm{N}$ - No to handshake, A - Avoid rumors. The novel coronavirus is a kind of disease that can be prevented with the right decision, discipline, and awareness. Hence, we must be disciplined and aware that we can overcome from this global crisis, prevent community transmission, and save our nation.

\section{REFERENCES}

1. India COVID-19 Tracker. Available from: https://www.covid19india. org.

2. Mattiuzzi C, Lippi G. Which lessons shall we learn from the 2019 novel coronavirus outbreak? Ann Transl Med 2020;8:48.

3. Coronavirus-Background, Symptoms and Treatment-Lesson Summary. Available from: https://www.alison.com/topic/learn/learningoutcomes.

4. Age, Sex, Existing Conditions of COVID-19 Cases and Deaths. 
Available from: https://www.worldometers.info/coronavirus/ coronavirus-age-sex-demographics. [Last accessed on 2020 Feb 29].

5. Xu H, Zhong L, Deng J, Peng J, Dan H, Zeng X, et al. High expression of ACE2 receptor of 2019-nCoV on the epithelial cells of oral mucosa. Int J Oral Sci 2020;12:8.

6. Chui P, Fukuoka J, Lourens RM, Taubenberger JK. Lung pathology of severe acute respiratory syndrome (SARS): A study of 8 autopsy cases from Singapore. Hum Pathol 2003;34:743-8.
7. Perlman S. Coronavirus: Novel Coronavirus (COVID-19) Infection. New York: Elsevier; 2020.

8. World Health Organization: Clinical Management of Severe Acute Respiratory Infection when Novel Coronavirus Disease (n-CoV) Infection is Suspected: Interim Guidance; 2020. Available from: https:// www.who.int/publications-detail/clinical-management-of-severeacute-respiratory-infection-when-novel-coronavirus-(ncov)-infectionis-suspected. 